\title{
Extra-Adrenal Silent Retroperitoneal Paraganglioma: A Rare Case
}

\author{
Dr. Swarjith Nimmakayala, Dr. Brijesh Kumar Sharma, Dr. Vikalap Gupta, \\ Department of General Surgery, Mahatma Gandhi Medical College, Jaipur, Rajasthan
}

\begin{abstract}
Extra- adrenal retroperitoneal paragangliomas are extremely rare neuroendocrine neoplasms with an incidence of 2-8 per million. They emanate from embryonic neural crest cells and are composed mainly of chromaffin cells located in the para- aortic sympathetic chain. They synthesize, store and secrete catecholamines due to which they may present with symptoms of hypertension like headache, sweating and palpitationand sometimes they may present with vague symptoms like pain abdomen and lump abdomen. On the other hand, they may remain silent and non-functional. Histopathologically and tumor marker wise, non-functional and the functional tumours are absolutely same. Primary methods of pre-operative diagnosis include imaging techniques which also help in surgical planning and pre-operative preparation of the patient. Non-functional tumours often escape preoperative diagnosis and create intraoperative complications. We present a case of non-functional extra-adrenal retroperitonealparaganglioma occurring in a 50-yearold male patient presenting with mass per abdomen. On Ultrasonongraphy, suspicion was towards a retroperitoneal mass of probable lymph nodal origin. On CT Scan, A large well defined heterogeneously enhancing mass lesion with solid and necrotic areas and few tiny foci of central calcifications is seen in left side of mesenteric measuring $11.4 \mathrm{~cm} * 11.7 \mathrm{~cm} * 9.6 \mathrm{~cm}(A P * T D * C C)$. No bowel invasion is seen. Prominent vessels are seen around the mass. IMP-Mass lesion left side of abdomen - likely mesenteric origin (? Malignant). The patient was posted for surgical resection of the mass. Per-operatively, the patient developed hypertensive crisis. Post operative period was uneventful and patient recovered well. This report highlights the importance of pre- operative diagnosis which is vital in the management of extra- adrenal retroperitoneal paraganglioma.
\end{abstract}

Keywords: Extra- Adrenal silent Retroperitoneal Paraganglioma

\section{Case Report}

A 50-year-old male patient presented to surgical department with complaints of lump in the left upper and mid abdomen. There was no history of vomiting or altered bowel habits or previous history of similar attacks. The patient was not a known hypertensive and was not on any medication.

Per abdominal examination revealed a mass in the left lumbar region extending into left hypochondriac region which did not move with respiration but side to side movement was present, globular in shape, rounded margins, smooth surface, firm in consistency, non ballotable. Bowel sounds and rectal examination were normal. Ultrasonography showed, a retroperitoneal mass of probable lymph nodal origin was suspected.On CECT. IMP- Mass lesion left side of abdomen - likely mesenteric origin(? Malignant).

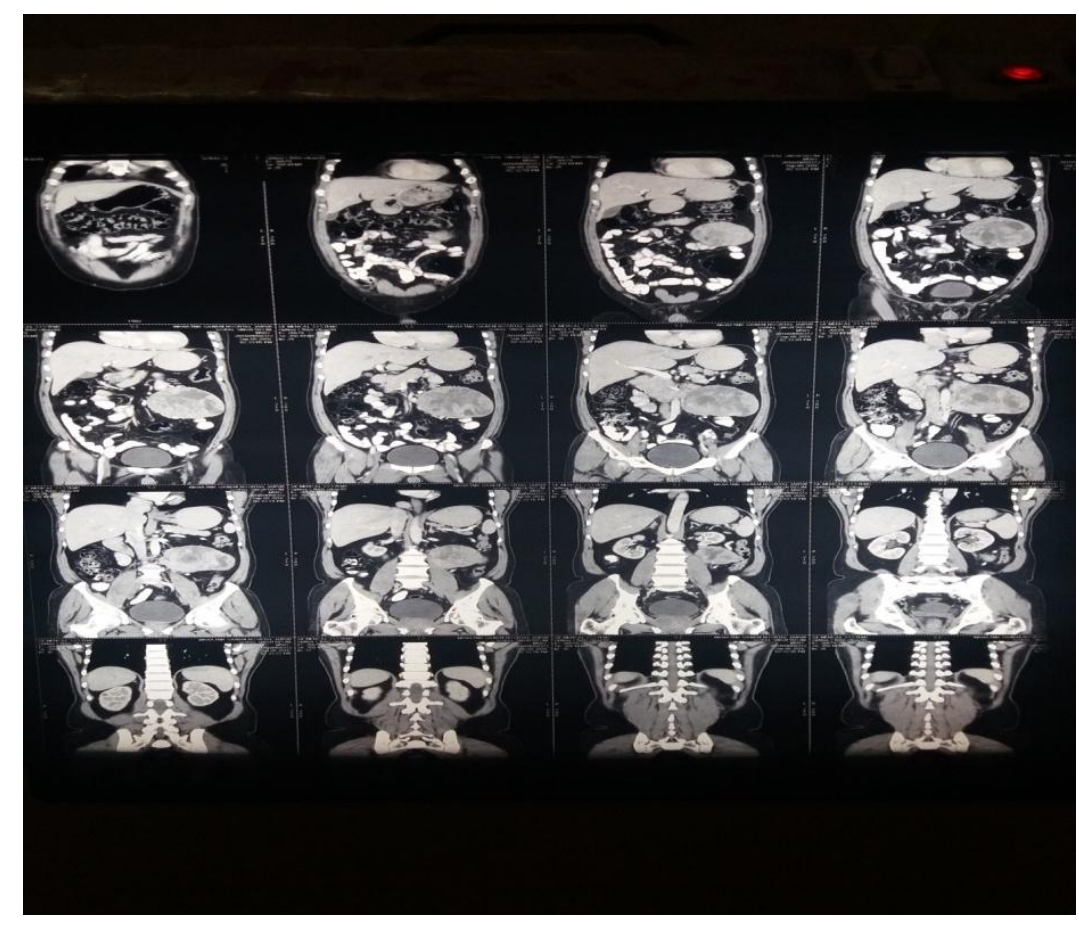

Volume 6 Issue 1, January 2017 www.ijsr.net 


\section{International Journal of Science and Research (IJSR) \\ ISSN (Online): 2319-7064}

Index Copernicus Value (2015): 78.96 | Impact Factor (2015): 6.391

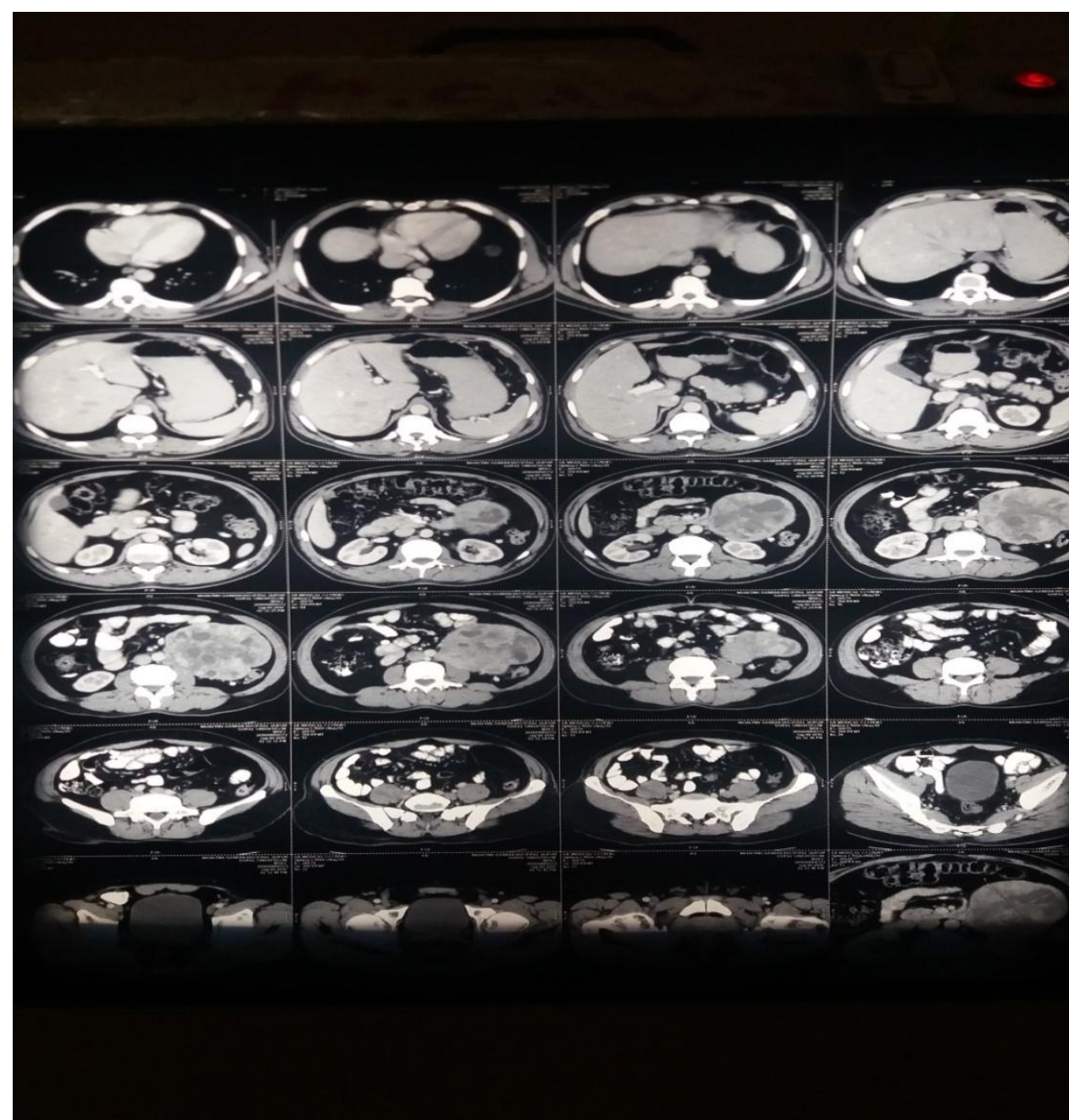

The hematological and biochemical profiles were normal. Laparotomy was undertaken for exploration by left paramedian incision. Per-operatively, a retroperitoneal mass was found which was highly vascular. The mass was found located anterior to the sympathetic and lymphatic chain. While attempting to separate the mass. Further dissection revealed that the mass was arising from the sympathetic chain. The mass was completely resected and was sent for histopathology.

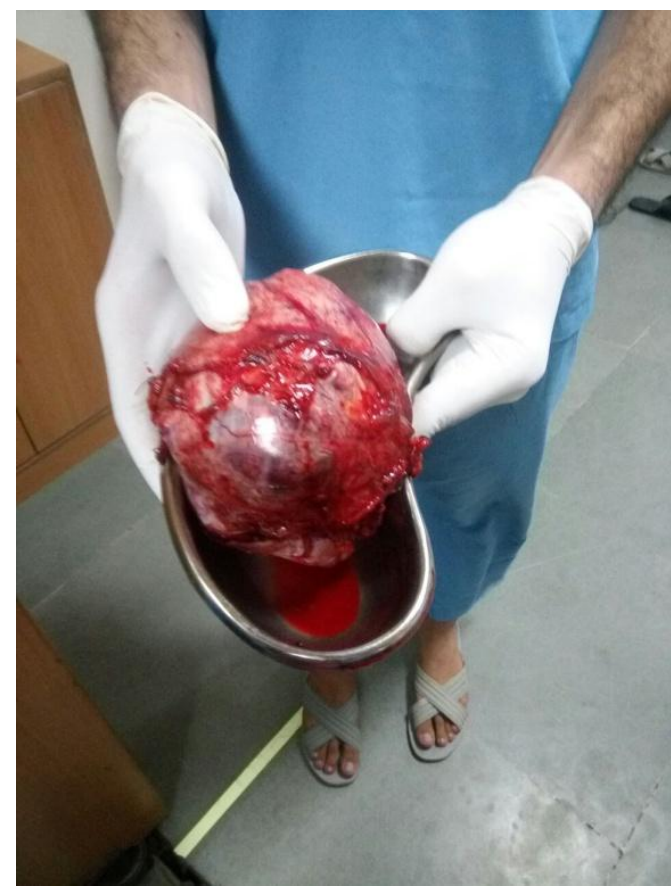

\section{Histopathological Findings}

Histopathology report says tumour made up of polygonal and spindle cells arranged in trabecular and solid patterns. The cells have abundant granular, amphophilic cytoplasm.

Volume 6 Issue 1, January 2017 www.ijsr.net 


\section{International Journal of Science and Research (IJSR) \\ ISSN (Online): 2319-7064 \\ Index Copernicus Value (2015): 78.96 | Impact Factor (2015): 6.391}

The cells show vesicular nuclei showing pleomorphism. The features are suggestive of extraadrenalparaganglioma
Histopathological diagnosis: Extra- adrenal retroperitoneal paraganglioma. Immunohistochemistry showed positivity for Chromogranin, Synaptophysin and S- 100 protein.

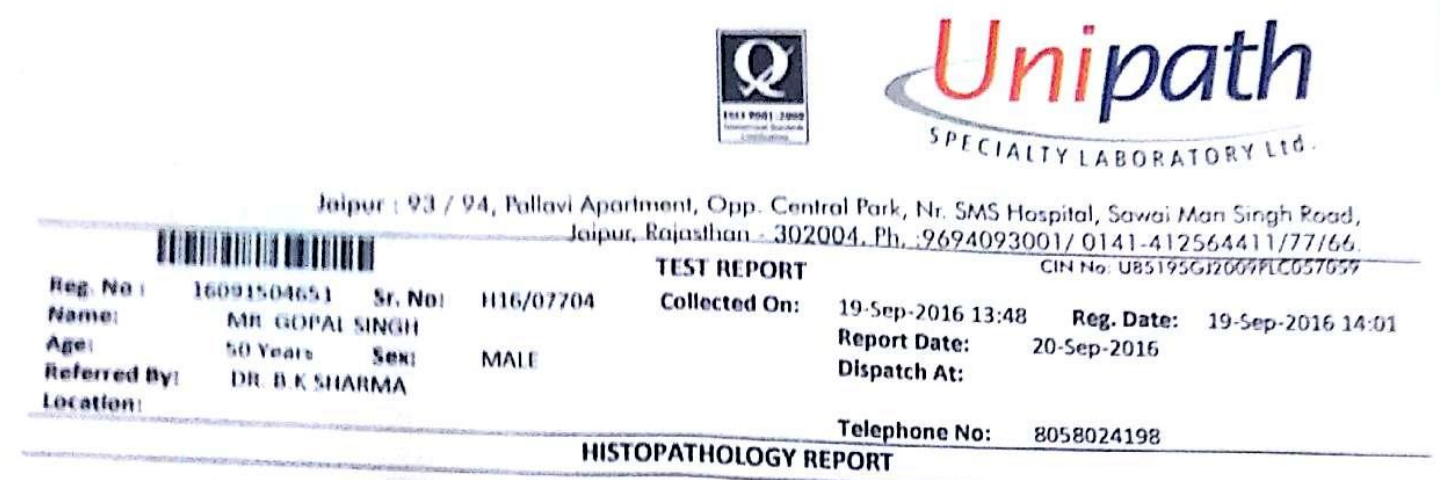

\section{Specimen}

Mesenteric lump. left lumbur region

\section{Gross Description}

Two paraffin block recelved, reference no. 091402/16

\section{Microscopic Description}

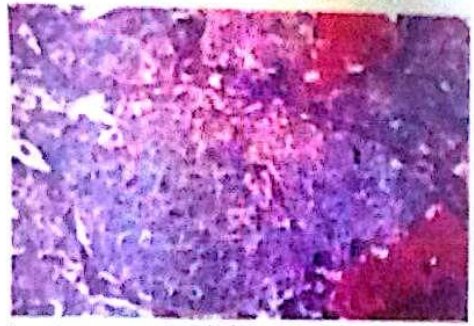

Section examined shows a tumor in nesting and trabecular pattern of cells within a prominent vascular network. Nests composed of polygonal colls and giant multinucleated cells with abundant granular eosinophilic or basophilic cytoplasm. The tumor cells have moderate nuclear pleomorphlsm and hyperchromasia, clear to eosinophilic cytoplasm. Rare mitosis (<1 mf/ $10 \mathrm{hpf}$ ) are seen.

\section{Immunohlstochemistry}

Chromogranin (Neuroendocrine marker) - Positive. Synaptophysin (Neuroendocrine marker) - Positive. S-100. positive

Ki-67(Proliferation Index)

$-<1 \%$

Approprlate internal and external controls are positive.

\section{Dlarnosis}

Extraadrenal Paraganglloma, left lumbur region.

Capsule-Intact. Mitotic activity-<1mf/10hpf.

All paraffin blocks labeled $\mathrm{H16/07704}$ returned along with report.

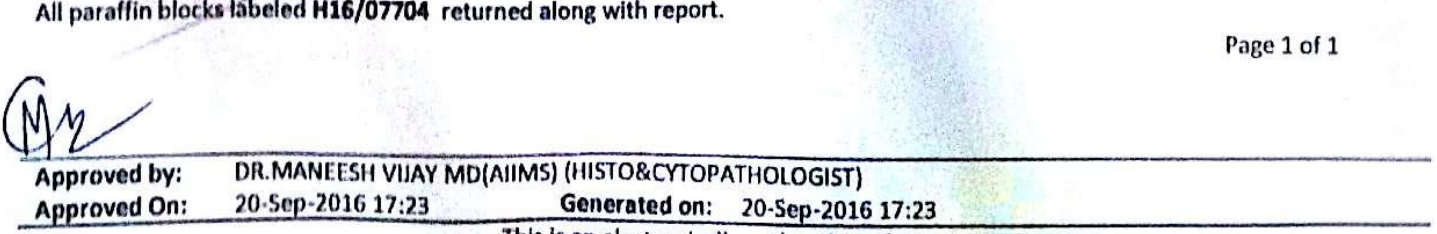

\section{Discussion}

Paragangliomas are rare neuroendocrine tumours emanating from specialized cells referred to as chief cells of paraganglia which are spread over the entire body ranging from the skull to the pelvic floor. Regardless of their location, these are referred to as "paragangliomas". The only exception to this rule being the paraganglioma arising from the adrenal medulla commonly referred to as "pheochromocytoma". The term "extra-

Volume 6 Issue 1, January 2017 


\section{International Journal of Science and Research (IJSR) \\ ISSN (Online): 2319-7064}

Index Copernicus Value (2015): 78.96 | Impact Factor (2015): 6.391

adrenalparaganglioma" is appropriate forparagangliomas situated outside adrenal medulla. Most of theseparagangliomas are known to occur in specific locations like the carotid body, jugular foramen, middle ear, aortico- pulmonary region, posterior mediastinum and abdominal para aortic region including the Zuckerkandl's body or aortic body. The adrenal medulla has the largest collection of receptor cells derived from the neural crest which accounts for the highest rate of occurrence of these tumours at this site. Whereas, extra- adrenal retroperitoneal paragangliomas emanate from the symmetrically distributed paraganglia situated in close relation to the aorta and sympathetic chain. These paraganglia which are histologically similar to carotid body and adrenal medullary paraganglia degenerate after the age of 1 to $1 \frac{1}{2}$ years.

Some of the paragangliomas are known to create, store and secrete catecholamines and are hence termed "functional paragangliomas". While these are diagnosed easily, nonfunctional paragangliomas may create major difficulties in pre-operative diagnosis.

Extra- adrenal paragangliomas are rarely encountered in daily clinical practice. If they are non- functional, they can pose additional diagnostic dilemmas thus causing difficulties in the choice of treatment and management approach.

Extra- adrenal retroperitoneal paragangliomas show classic histological features which help in reaching the diagnosis. However, the correct management lies in the pre-operative diagnosis since pre-operative pharmacological preparation with alpha- adrenergic blockade for 2- 4 weeks to prevent and treat a syndrome of possible intra- operative catecholamine release may be started failing which, catecholamine induced complications like hypertensive crisis, cardiac arrhythmias, pulmonary oedema and cardiac ischemia due to manipulation of the tumour may arise. Even a hypotensive crisis may occur after tumour removal. Preoperative diagnostic modalities include radio- imaging techniques comprising USG, CT, MRI, I 131, MIBG and octreotide along with endocrine secretion evaluations. The tumour was diagnosed intra-operatively based on its classic shooting of the blood pressure on manipulating it and sudden fall in blood pressure on its complete removal which was further confirmed post operatively on the basis of histologic features and tumour markers.

A number of factors contribute to the difficulties encountered in the management of extra- adrenal paragangliomas. They include the rarity of extra- adrenal paragangliomas which account for just 2-8 per million incidence, the insufficiency of information available regarding their natural history and outcome and the nonavailability of a definitive pre- operative diagnosis especially in the case of a non- functional tumour.

Extra- adrenal paragangliomas can be uni or multicentric with a tendency for local invasion and therefore a thorough evaluation needs to be undertaken to rule out multicentricity. Periodic follow- ups will have to be scheduled to rule out recurrence. 24- $50 \%$ of extra- adrenal paragangliomas are malignant in contrast to malignant pheochromocytomas which have an incidence of $10 \%$. The tumour in the present case was benign, unicentric and lacked local invasion.

Wendelin et al., observed that benign and malignant lesions could not be distinguished based on histopathological findings alone. They gave the opinion that distant metastases and local invasion of adjacent organs were the only reliable indicators of malignancy. The tumour in the present case was interpreted as benign based on the histopathological findings.

Sclafani et al., concluded that patients in whom a complete surgical dissection was possible had a better overall survival than patients in whom it was not. Complete surgical resection was possible in the present case.

Malignant extra- adrenal paragangliomas are known to metastasize to bone, liver, peritoneum, pelvis, ovaries, cervical lymph nodes and lung.

The pre-operative differential diagnosis considered in the present case were retroperitoneal malignant mesenchymal tumour and Lymph nodal mass.

\section{Conclusion}

Extra- adrenal retroperitoneal paragangliomas are rare neoplasms which may cause difficulty in diagnosis and treatment. Yet, a pre-operative diagnosis is crucial for preoperative preparation and proper planning of management.Therefore, we wish to highlight the importance of pre- operative diagnosis in these tumours due to our own experience of a non- functional tumour which escaped a definitive pre- operative diagnosis resulting in unforeseen development of per operative hypertensive crisis.

\section{References}

[1] Feng N, Zhang WY, Wu XT. Clinicopathological analysis of paraganglioma with literature review. World J Gastroenterol. 2009;15:3003-08.

[2] Bismar HA, Murshid KR. Extra adrenal retroperitoneal paraganglioma. Saudi Med J. 2003;24:778-80.

[3] Wen J, Li HZ, Ji ZG, Mao QZ, Shi BB, et al. A decade of clinical experience with extra- adrenal paragangliomas of retroperitoneum: Report of 67 cases and a literature review. Urol Ann. 2010;2:12-16.

[4] Hayes WS, Davidson AJ, Grimley PM, Hartman DS. Extra- adrenal retroperitoneal paraganglioma: clinical, pathologic and CT findings. Am J Rad. 1990;155:124750 .

[5] Sclafani LM, Woodruff JM, Murray FB. Extraadrenal retroperitoneal paragangliomas- from the departments of Surgery and Pathology, Memorial Sloan- Kettering Cancer center, New York. Surgery.1990;108:1124-30. 